\title{
Estimating vaccine effectiveness against laboratory-confirmed influenza among children and adolescents in Lower Saxony and Saxony-Anhalt, 2012-2016
}

\author{
A. MÖHL ${ }^{1 *}$, L. GRÄFE ${ }^{2}$, C. $\mathrm{HELMEKE}^{2}$, D. ZIEHM ${ }^{1}$, M. MONAZAHIAN ${ }^{1}$, \\ H.-M. IRMSCHER ${ }^{2}$ AND J. DREESMAN ${ }^{1}$ \\ ${ }^{1}$ Governmental Institute of Public Health of Lower Saxony, Hannover, Lower Saxony, Germany \\ ${ }^{2}$ State Agency for Consumer Protection of Saxony-Anhalt, Magdeburg, Saxony-Anhalt, Germany
}

Received 31 March 2017; Final revision 4 November 2017; Accepted 6 November 2017; first published online 6 December 2017

\section{SUMMARY}

Influenza vaccine effectiveness (VE) has to be estimated anew for every season to explore vaccines' protective effect in the population. We report VE estimates against laboratoryconfirmed influenza $\mathrm{A}(\mathrm{H} 1 \mathrm{~N} 1)$ pdm09, $\mathrm{A}(\mathrm{H} 3 \mathrm{~N} 2)$ and influenza B among children aged 2-17 years, using test-negative design. Pooled data from two German federal states' surveillance systems for acute respiratory illness from week 40/2012 to 20/2016 was used, yielding a total of 10627 specimens. Odds ratios and 95\% confidence intervals (95\% CIs) for the association between laboratory-confirmed influenza and vaccination status were calculated by multivariate logistic regression adjusting for age, sex, illness onset and federal state. VE was estimated as 1-Odds Ratio. Overall adjusted VE was 33\% (95\% CI: 24-3-40.7). A strong variation of VE between the seasons and subtypes was observed: highest season- and subtype-specific VE of $86 \cdot 2 \%$ (95\% CI: 41.3-96.7) was found against $\mathrm{A}(\mathrm{H} 1 \mathrm{~N} 1) \mathrm{pdm} 09$ in 7-17-year-olds in 2015/16. Low estimates of VE were observed against $\mathrm{A}(\mathrm{H} 3 \mathrm{~N} 2)$ in any season, e.g. 1.5\% (95\% CI: -39·3-30.3) in 2014/15. Estimates showed a tendency to higher VE among 7-17-year-old children, but differences were not statistically significant. Although our findings are common in studies estimating influenza VE, we discussed several explanations for observed low VE.

Key words: Epidemiology, influenza (seasonal), routine surveillance data, test-negative design, vaccine effectiveness.

\section{INTRODUCTION}

According to estimates for the global burden of seasonal influenza by the World Health Organization (WHO), influenza causes approximately one billion cases and $300000-500000$ of deaths annually [1].

In Germany, annual vaccination against influenza is recommended for persons older than 60 years. Furthermore, vaccination is recommended by indication

\footnotetext{
* Author for correspondence: Annika Möhl, Wilde Heide 4, 21220 Seevetal, Germany.

(Email: annikamoehl@freenet.de)
}

for the following risk groups: women in the second or third trimester of pregnancy, people living in nursing homes, persons with high occupational risk (e.g. health personnel) and people suffering from chronic diseases (e.g. asthma, cardiovascular disease, diabetes mellitus, HIV) including children with such conditions [2]. Despite these recommendations, influenza vaccination of healthy children is in most cases also covered by medical insurance companies in Saxony-Anhalt and Lower Saxony.

According to the German vaccination recommendations during the years 2013/14 to 2015/ 
16 , children aged 2-6 years with an indication for seasonal influenza vaccination should preferably receive live attenuated influenza vaccine (LAIV) instead of inactivated influenza vaccines (IIV). Children with chronic diseases aged 7-17 years could either receive LAIV or IIV [2, 3]. Preferential recommendation of LAIV was cancelled in 2016/17 [4]. Initially, a meta-analysis from Falkenhorst et al., comparing the efficacy of LAIV and IIV, showed a higher efficacy of LAIV than of IIV in 2-6-year-old children [5]. However, observational study results from the recent seasons indicated a weak preventive effect of LAIV, especially against influenza $\mathrm{A}(\mathrm{H} 1 \mathrm{~N} 1) \operatorname{pdm} 09[4,6]$.

Due to antigenic drift of influenza viruses, vaccine composition has to be adapted annually in order to match circulating viruses and is recommended by the WHO [7-11].

The efficacy of a vaccine is firstly tested under controlled conditions, such as randomised controlled trials (RCT), before a new vaccine gets licensed [12]. For ethical and economic reasons, such trials are not feasible for influenza because influenza vaccine formulation changes annually.

In contrast, vaccine effectiveness (VE) measures the reduction of a disease in the field under ordinary conditions [12]. A commonly used method to estimate the effectiveness of influenza vaccines in the field annually and quickly is the test-negative design (TND) [13-18].

There are several possible factors that could have an impact on influenza VE such as possible changes of the circulating viruses and the composition of the vaccine itself or characteristics of the study population.

Estimating and monitoring VE is important to explore vaccines' protective effect in the target population under ordinary conditions [13]. It may help to recognise a lack of protection and could be helpful when adapting vaccination recommendations and redefining the target population.

This study aims to estimate VE against laboratoryconfirmed influenza among 2-17-year-old children and adolescents in two German federal states during four influenza seasons from 2012 to 2016. Therefore, pooled data from both states' virological surveillance systems for acute respiratory illness (ARI) of the Governmental Institute of Public Health in Lower Saxony and the State Agency for Consumer Protection in Saxony-Anhalt was used. We pooled data of these states because of the similar surveillance systems for ARI and a long-term cooperation with the aim of increasing sample size for influenza VE estimation.

\section{METHODS}

\section{Study population and study design}

We analysed data of 2-17-year-old children from the ARI-sentinel systems of Lower Saxony and Saxony-Anhalt over four consecutive influenza seasons from 2012/13 to 2015/16, each monitoring season lasting from calendar weeks 40 to 20 of the following year.

In Lower-Saxony, 40 general practitioners, predominantly paediatricians and four hospitals participate in the influenza sentinel surveillance system. Samples from nasopharynx of children presenting ARI, defined as pharyngitis, bronchitis or pneumonia with or without fever, are taken from a maximum of seven patients per week by general practitioners and of 15 patients per week by hospitals. Samples are analysed in the laboratory of the Governmental Institute of Public Health of Lower Saxony. The sentinel system from Saxony-Anhalt consists of 15 paediatricians. The procedure of sampling and analysing is similar to the procedure of Lower Saxony and is described in detail elsewhere [19].

The laboratories use real-time reverse transcription polymerase chain reaction (RT-PCR) to test for influenza and other viruses causing acute respiratory symptoms. In addition, practitioners complete a questionnaire, which provides basic demographic data, information on symptoms, on date of illness onset and on vaccination of the respective patient.

The present study comprises 2-17-year-old patients swabbed from nasopharynx. Patients with missing values of vaccination status or vaccinated within 14 days before illness onset were excluded from data analysis as well as patients with missing data on sex or age.

We also excluded data of patients with an onset of illness more than 8 days before the date of sample collection since the viral load, and thus the probability of detecting the virus, decreases over time [20].

If the date of illness onset was missing, it was imputed by 'sampling date minus median-difference between sampling date and illness onset' during the particular season.

We considered a patient as vaccinated when she or he had received at least one dose of any influenza vaccine recommended by the WHO and approved in Germany for the particular season. A case was defined as a patient tested positive for at least one of the influenza virus subtypes $\mathrm{A}(\mathrm{H} 1 \mathrm{~N} 1) \mathrm{pdm} 09$, A (H3N2) or influenza B. Cases tested positive for 
unsubtyped influenza A were included when estimating VE against any influenza, but excluded for the subtype analyses. When estimating overall influenza $\mathrm{VE}$, a case was counted only once, even if tested positive for more than one subtype (co-infection). In the subtype-analyses, patients with co-infections were considered as a case for every subtype they tested positive for. Controls had to be tested negative for all influenza virus subtypes but could be tested positive for other viruses causing ARI, e.g. picornavirus or adenovirus. Consecutive testing of the same patient could not be identified and was considered as a new participant for every test.

\section{Laboratory methods}

Samples of patients with acute onset of respiratory symptoms were analysed qualitatively using RTPCR to detect RNA of influenza A and B. RTPCR was conducted in a LightCycler 480 (Roche, Mannheim, Germany) in Lower Saxony and in a Rotor-Gene (Corbett) in Saxony-Anhalt using specific primers and probes covering the matrix gene region of influenza A- and B-genome. A second assay for the subtype-independent detection of influenza A viruses was supplemented with a set of PCR systems that allowed differentiation of haemagglutinin and neuraminidase subtypes including influenza $\mathrm{A}(\mathrm{H} 1 \mathrm{~N} 1)$ pdm09 and $\mathrm{A}(\mathrm{H} 3 \mathrm{~N} 2)$ viruses. Positive samples were sent to the National Reference Centre for Influenza at the Robert Koch-Institute (Berlin, Germany) for sequence analyses and nationwide influenza surveillance.

\section{Statistical analysis}

We used TND for estimation of influenza VE. We estimated the association of influenza virus confirmation with vaccination status via Odds ratios $(\mathrm{OR})$ calculated by using multivariate logistic regression adjusting for age, sex, month of illness onset and federal state. For estimating VE against laboratoryconfirmed influenza, we used the formula 1-OR. VE was considered statistically significant when $95 \%$ confidence limits $(95 \%$ CIs) excluded zero.

Separate analyses were conducted for influenza virus subtypes (A(H1N1)pdm09, $\mathrm{A}(\mathrm{H} 3 \mathrm{~N} 2)$ and influenza virus $\mathrm{B}$ ) and for any influenza virus. The analyses were stratified for two age groups (2-6 years and 7-17 years). VE was reported if 30 or more cases of influenza occurred.
We did not conduct separate analyses for types of vaccine (LAIV vs. IIV) since information about the used type of vaccine has been collected for Lower Saxony only since 2016.

In order to characterise influenza seasons, seasonspecific influenza positive proportions were calculated as the number of patients tested positive for influenza divided by the number of all tested patients. We conducted sensitivity analysis for VE in 2015/16, excluding children with missing date of illness onset instead of imputing the date.

Statistical analyses were conducted using IBM SPSS Statistics for Windows, Version 23.0 (IBM Corp. Released 2015. Armonk, NY).

\section{Ethical aspects}

Samples and questionnaires were collected for the purpose of routine virological sentinel surveillance by the State Health Agencies of Lower Saxony and Saxony-Anhalt to monitor the course and severity of ARI caused by different viruses, including influenza virus. For the analysis of VE, data were completely anonymised so that patients could not be identified. Since VE analysis was a secondary data analysis based on routine surveillance data, ethical approval was not regarded as necessary [21].

\section{RESULTS}

\section{Description of the study population and influenza seasons}

Between 28 September 2012 and 28 May 2016, 11316 samples of children aged 2-17 years were analysed within the monitoring systems of Lower Saxony and Saxony-Anhalt.

We excluded 689 specimens that did not meet the inclusion criteria (e.g. missing information on vaccination status or sex) which yielded a final dataset of 10627 specimens with $7333(69 \cdot 0 \%)$ samples from Lower Saxony and $3294 \quad(31.0 \%)$ from Saxony-Anhalt (Table 1).

A total of $5644(53 \cdot 1 \%)$ patients were male and the overall median age was 5.6 years (mean age $7 \cdot 0$ years). Of all patients, $6245(58 \cdot 8 \%)$ were aged $2-6$ years and $4382(41 \cdot 2 \%)$ belonged to age group $7-17$ years (Table 1). Across all seasons, $7 \cdot 5 \%$ of all patients and $8.1 \%$ of the controls were vaccinated. The latter group can be regarded as an estimate for the population seeking health care. In this group, the seasonal 
Table 1. Characteristics of the study population

\begin{tabular}{llll}
\hline \hline Variable & Cases $(\%), n=3304$ & Controls $(\%), n=7323$ & Total $(\%), N=10627$ \\
\hline $\begin{array}{l}\text { Federal state } \\
\text { Lower Saxony }\end{array}$ & $2319(70 \cdot 2)$ & & \\
$\quad$ Saxony-Anhalt & $985(29 \cdot 8)$ & $5014(68 \cdot 5)$ & $7333(69 \cdot 0)$ \\
Sex & & $2309(31 \cdot 5)$ & $3294(31 \cdot 0)$ \\
$\quad$ Male & $1788(54 \cdot 1)$ & $3856(52 \cdot 7)$ & $5644(53 \cdot 1)$ \\
Female & $1516(45 \cdot 9)$ & $3467(47 \cdot 3)$ & $4983(46 \cdot 9)$ \\
Age group & & & \\
2-6 & $1725(52 \cdot 2)$ & $4520(61 \cdot 7)$ & $6245(58 \cdot 8)$ \\
$7-17$ & $1579(47 \cdot 8)$ & $2803(38 \cdot 3)$ & $4382(41 \cdot 2)$ \\
Vaccinated & & & \\
Yes & $209(6 \cdot 3)$ & $591(8 \cdot 1)$ & $800(7 \cdot 5)$ \\
No & $3095(93 \cdot 7)$ & $6732(91 \cdot 9)$ & $9827(92 \cdot 5)$ \\
Disease onset & & & \\
Sept & $0(0 \cdot 0)$ & $26(0 \cdot 4)$ & $723(0 \cdot 2)$ \\
Oct & $3(0 \cdot 1)$ & $720(9 \cdot 8)$ & $914(8 \cdot 6)$ \\
Nov & $40(1 \cdot 2)$ & $874(11 \cdot 9)$ & $877(8 \cdot 3)$ \\
Dec & $116(3 \cdot 5)$ & $761(10 \cdot 4)$ & $1959(18 \cdot 4)$ \\
Jan & $629(19 \cdot 0)$ & $1330(18 \cdot 2)$ & $2815(26 \cdot 5)$ \\
Feb & $1323(40 \cdot 0)$ & $1492(20 \cdot 4)$ & $2278(21 \cdot 4)$ \\
Mar & $947(28 \cdot 7)$ & $1331(18 \cdot 2)$ & $851(8 \cdot 0)$ \\
Apr & $231(7 \cdot 0)$ & $620(8 \cdot 5)$ & $184(1 \cdot 7)$ \\
May & $15(0 \cdot 5)$ & $169(2 \cdot 3)$ & \\
\hline \hline
\end{tabular}

Table 2. Vaccination coverage, 2012-2016

\begin{tabular}{|c|c|c|c|c|c|c|}
\hline \multirow[b]{2}{*}{ Season } & \multicolumn{6}{|c|}{ Vaccination coverage* } \\
\hline & Total & $\begin{array}{l}\text { Cases } \\
\text { Vaccin. }(\%)^{*}\end{array}$ & Total & $\begin{array}{l}\text { Controls } \\
\text { Vaccin. }(\%)\end{array}$ & Total & $\begin{array}{l}\text { Total } \\
\text { Vaccin. (\%) }\end{array}$ \\
\hline $2012 / 13$ & 1443 & $67(4 \cdot 6)$ & 1792 & $146(8 \cdot 1)$ & 3235 & $213(6 \cdot 6)$ \\
\hline 2-6-year-olds & 724 & $33(4 \cdot 6)$ & 1149 & $76(6 \cdot 6)$ & 1873 & $109(5 \cdot 8)$ \\
\hline 7-17-year-olds & 719 & $34(4 \cdot 7)$ & 643 & $70(10 \cdot 9)$ & 1362 & $104(7 \cdot 6)$ \\
\hline $2013 / 14$ & 152 & $16(10 \cdot 5)$ & 1867 & $144(7 \cdot 7)$ & 2019 & $160(7 \cdot 9)$ \\
\hline 2-6-year-olds & 95 & $9(9 \cdot 5)$ & 1174 & $75(6 \cdot 4)$ & 1269 & $84(6 \cdot 6)$ \\
\hline 7-17-year-olds & 57 & $7(12 \cdot 3)$ & 693 & $69(10 \cdot 0)$ & 750 & $76(10 \cdot 1)$ \\
\hline $2014 / 15$ & 940 & $82(8 \cdot 7)$ & 2071 & $179(8 \cdot 6)$ & 3011 & $261(8 \cdot 7)$ \\
\hline 2-6-year-olds & 495 & $34(6 \cdot 9)$ & 1225 & $93(7 \cdot 6)$ & 1720 & $127(7 \cdot 4)$ \\
\hline 7-17-year-olds & 445 & $48(10 \cdot 8)$ & 846 & $86(10 \cdot 2)$ & 1291 & $134(10 \cdot 4)$ \\
\hline $2015 / 16$ & 769 & $44(5 \cdot 7)$ & 1593 & $122(7 \cdot 7)$ & 2362 & $166(7 \cdot 0)$ \\
\hline 2-6-year-olds & 411 & $22(5 \cdot 4)$ & 972 & $63(6 \cdot 5)$ & 1383 & $85(6 \cdot 1)$ \\
\hline 7-17-year-olds & 358 & $22(6 \cdot 1)$ & 621 & $59(9 \cdot 5)$ & 979 & $81(8 \cdot 3)$ \\
\hline 2012-2016 & 3304 & $209(6 \cdot 3)$ & 7323 & $591(8 \cdot 1)$ & 10627 & $800(7 \cdot 5)$ \\
\hline 2-6-year-olds & 1725 & $98(5 \cdot 7)$ & 4520 & $307(6 \cdot 8)$ & 6245 & $405(6 \cdot 5)$ \\
\hline 7-17-year-olds & 1579 & $111(7 \cdot 0)$ & 2803 & $284(10 \cdot 1)$ & 4382 & $395(9 \cdot 0)$ \\
\hline
\end{tabular}

* Number (percentage) of vaccinated patients.

coverages varied slightly between $7 \cdot 7 \%$ and $8 \cdot 6 \%$, not showing a substantial trend. Vaccination coverage was slightly higher in 7-17-year-olds than in the younger ones. Season-, age group- and group-specific vaccination coverage is shown in Table 2.
The majority of samples (7052 of $10627,66 \cdot 4 \%$ ) was taken between January and March.

In total, $3304(31 \cdot 1 \%)$ patients tested positive for influenza. Cases were slightly older than controls (median age 6.5 years vs. 5.5 years). Among all 
Table 3. Estimated vaccine effectiveness (VE) against laboratory-confirmed influenza (all subtypes) and influenza A(H1N1)pdm09 stratified for age groups, $2012-2016$

\begin{tabular}{|c|c|c|c|c|c|c|c|c|c|}
\hline \multirow[b]{2}{*}{ Season } & \multirow[b]{2}{*}{$\begin{array}{l}\text { Age } \\
\text { group }\end{array}$} & \multicolumn{4}{|c|}{ Any influenza } & \multicolumn{4}{|c|}{$\mathrm{A}(\mathrm{H} 1 \mathrm{~N} 1) \mathrm{pdm} 09$} \\
\hline & & $\begin{array}{l}\text { Cases } \\
\text { (vaccin.) }\end{array}$ & $\begin{array}{l}\text { Controls } \\
\text { (vaccin.) }\end{array}$ & $\begin{array}{l}\text { Crude VE in \% } \\
(95 \% \text { CI })\end{array}$ & $\begin{array}{l}\text { Adjusted* } \\
\text { VE in } \%(95 \% \text { CI })\end{array}$ & $\begin{array}{l}\text { Cases } \\
\text { (vaccin.) }\end{array}$ & $\begin{array}{l}\text { Controls } \\
\text { (vaccin.) }\end{array}$ & $\begin{array}{l}\text { Crude VE in \% } \\
(95 \% \mathrm{CI})\end{array}$ & $\begin{array}{l}\text { Adjusted* } \\
\text { VE in } \%(95 \% \text { CI })\end{array}$ \\
\hline \multirow[t]{3}{*}{$2012 / 13$} & $2-17$ & $1443(67)$ & $1792(146)$ & $45 \cdot 1(26 \cdot 1-59 \cdot 2)$ & $50 \cdot 7(32 \cdot 5-64 \cdot 0)$ & $278(11)$ & $1792(146)$ & $53 \cdot 6(13 \cdot 1-75 \cdot 2)$ & $56 \cdot 7(17 \cdot 1-77 \cdot 4)$ \\
\hline & $2-6$ & $724(33)$ & $1149(76)$ & $32 \cdot 6(-2 \cdot 6-55 \cdot 7)$ & $37 \cdot 1(2 \cdot 2-59 \cdot 6)$ & $164(7)$ & $1149(76)$ & $37 \cdot 1(-39 \cdot 0-71 \cdot 5)$ & $33 \cdot 1(-52 \cdot 8-70 \cdot 7)$ \\
\hline & $7-17$ & $719(34)$ & $643(70)$ & $59.4(37.9-73.4)$ & $57 \cdot 8(33 \cdot 5-73 \cdot 2)$ & $114(4)$ & $643(70)$ & $70 \cdot 2(16 \cdot 8-89 \cdot 4)$ & $73 \cdot 2(22 \cdot 7-90 \cdot 7)$ \\
\hline \multirow{3}{*}{$2013 / 14$} & $2-17$ & $152(16)$ & $1867(144)$ & $-40 \cdot 8(-142 \cdot 8-18 \cdot 4)$ & $-4 \cdot 3(-2 \cdot 9-40 \cdot 5)$ & $27(1)$ & $1867(144)$ & $\dagger$ & $\dagger$ \\
\hline & $2-6$ & $95(9)$ & $1174(75)$ & $-53 \cdot 3(-216 \cdot 8-25 \cdot 8)$ & $-25 \cdot 5(-165 \cdot 5-40 \cdot 7)$ & $18(1)$ & $1174(75)$ & $\dagger$ & $\dagger$ \\
\hline & $7-17$ & $57(7)$ & $693(69)$ & $-26 \cdot 6(-190 \cdot 1-44 \cdot 7)$ & $8 \cdot 8(-114 \cdot 0-61 \cdot 2)$ & $9(0)$ & $693(69)$ & $\dagger$ & $\dagger$ \\
\hline \multirow[t]{3}{*}{$2014 / 15$} & $2-17$ & $940(82)$ & $2071(179)$ & $-1 \cdot 0(-32 \cdot 8-23 \cdot 2)$ & $22 \cdot 3(-4 \cdot 7-42 \cdot 3)$ & $182(15)$ & $2071(179)$ & $5 \cdot 1(-64 \cdot 6-45 \cdot 2)$ & $9 \cdot 3(-62 \cdot 0-49 \cdot 3)$ \\
\hline & $2-6$ & $495(34)$ & $1225(93)$ & $10 \cdot 2(-34 \cdot 9-40 \cdot 3)$ & $22 \cdot 7(-21 \cdot 8-50 \cdot 9)$ & $133(9)$ & $1225(93)$ & $11 \cdot 7(-79 \cdot 5-56 \cdot 5)$ & $21 \cdot 3(-67 \cdot 7-63 \cdot 0)$ \\
\hline & $7-17$ & $445(48)$ & $846(86)$ & $-6 \cdot 8(-55 \cdot 2-26 \cdot 4)$ & $21 \cdot 6(-17 \cdot 0-47 \cdot 5)$ & $49(6)$ & $846(86)$ & $-23 \cdot 3(-198 \cdot 1-49 \cdot 0)$ & $-8 \cdot 0(-173 \cdot 0-57 \cdot 3)$ \\
\hline \multirow[t]{3}{*}{$2015 / 16$} & $2-17$ & $769(44)$ & $1593(122)$ & $26 \cdot 8(-4 \cdot 5-48 \cdot 7)$ & $49 \cdot 4(25 \cdot 7-65 \cdot 5)$ & $324(15)$ & $1593(122)$ & $41 \cdot 5(-1 \cdot 5-66 \cdot 2)$ & $60 \cdot 2(29 \cdot 3-77 \cdot 6)$ \\
\hline & $2-6$ & $411(22)$ & $972(63)$ & $18 \cdot 4(-34 \cdot 5-50 \cdot 5)$ & $44 \cdot 8(4 \cdot 6-68 \cdot 0)$ & $219(13)$ & $972(63)$ & $8 \cdot 9(-68 \cdot 6-50 \cdot 8)$ & $44 \cdot 8(-6 \cdot 9-71 \cdot 5)$ \\
\hline & $7-17$ & $358(22)$ & $621(59)$ & $37 \cdot 6(-3 \cdot 6-62 \cdot 5)$ & $52 \cdot 9(18 \cdot 4-72 \cdot 8)$ & $105(2)$ & $621(59)$ & $81 \cdot 5(23 \cdot 1-95 \cdot 6)$ & $86 \cdot 2(41 \cdot 3-96 \cdot 7)$ \\
\hline \multirow[t]{3}{*}{$2012-2016$} & $2-17$ & $3304(209)$ & $7323(591)$ & $23 \cdot 1(9 \cdot 4-34 \cdot 7)$ & $33 \cdot 0(24 \cdot 3-40 \cdot 7)$ & $811(42)$ & $7323(591)$ & $37 \cdot 8(14 \cdot 2-54 \cdot 9)$ & $47 \cdot 0(25 \cdot 7-62 \cdot 2)$ \\
\hline & $2-6$ & $1725(98)$ & $4520(307)$ & $17 \cdot 3(-4 \cdot 5-34 \cdot 6)$ & $27 \cdot 8(6 \cdot 8-44 \cdot 1)$ & $534(30)$ & $4520(307)$ & $18 \cdot 3(-20 \cdot 2-44 \cdot 5)$ & $34 \cdot 6(1 \cdot 0-56 \cdot 8)$ \\
\hline & $7-17$ & $1579(111)$ & $2803(284)$ & $32 \cdot 9(15 \cdot 7-46 \cdot 6)$ & $40 \cdot 1(23 \cdot 0-53 \cdot 4)$ & $277(12)$ & $2803(284)$ & $59 \cdot 8(27 \cdot 4-77 \cdot 8)$ & $63 \cdot 6(33 \cdot 1-80 \cdot 3)$ \\
\hline
\end{tabular}

Statisically significant estimates (95\% confidence limits excluding zero) are printed in bold.

* Adjusted for sex, age, month of illness onset and federal state.

$\dagger$ No VE estimate because of a too small number of cases $(<30)$.

\$ Adjusted estimates additionally adjusted for season. 
Table 4. Estimated vaccine effectiveness (VE) against laboratory-confirmed influenza A(H3N2) and influenza B stratified for age groups, 2012-2016

\begin{tabular}{|c|c|c|c|c|c|c|c|c|c|}
\hline \multirow[b]{2}{*}{ Season } & \multirow[b]{2}{*}{$\begin{array}{l}\text { Age } \\
\text { group }\end{array}$} & \multicolumn{4}{|l|}{$\mathrm{A}(\mathrm{H} 3 \mathrm{~N} 2)$} & \multicolumn{4}{|l|}{ B } \\
\hline & & $\begin{array}{l}\text { Cases } \\
\text { (vaccin.) }\end{array}$ & $\begin{array}{l}\text { Controls } \\
\text { (vaccin.) }\end{array}$ & $\begin{array}{l}\text { Crude VE in \% } \\
(95 \% \mathrm{CI})\end{array}$ & $\begin{array}{l}\text { Adjusted* } \\
\text { VE in } \%(95 \% \mathrm{CI})\end{array}$ & $\begin{array}{l}\text { Cases } \\
\text { (vaccin.) }\end{array}$ & $\begin{array}{l}\text { Controls } \\
\text { (vaccin.) }\end{array}$ & $\begin{array}{l}\text { Crude VE in \% } \\
(95 \% \mathrm{CI})\end{array}$ & $\begin{array}{l}\text { Adjusted* } \\
\text { VE in } \%(95 \% \mathrm{CI})\end{array}$ \\
\hline \multirow[t]{3}{*}{$2012 / 13$} & $2-17$ & $472(30)$ & $1792(146)$ & $23 \cdot 5(-14 \cdot 9-49 \cdot 0)$ & $30 \cdot 2(-7 \cdot 1-54 \cdot 5)$ & $696(26)$ & $1792(146)$ & $56 \cdot 3(33 \cdot 0-71 \cdot 4)$ & $58 \cdot 6(34 \cdot 0-74 \cdot 0)$ \\
\hline & $2-6$ & $307(15)$ & $1149(76)$ & $27 \cdot 5(-28 \cdot 1-$ & $29 \cdot 7(-27 \cdot 9-61 \cdot 4)$ & $256(11)$ & $1149(76)$ & $36 \cdot 6(-21 \cdot 1-66 \cdot 8)$ & $41 \cdot 1(-18 \cdot 5-70 \cdot 7)$ \\
\hline & $7-17$ & $165(15)$ & $643(70)$ & $18 \cdot 1(-47 \cdot 1-54 \cdot 4)$ & $23 \cdot 3(-43 \cdot 6-59 \cdot 1)$ & $440(15)$ & $643(70)$ & $71 \cdot 1(48 \cdot 8-83 \cdot 7)$ & $65 \cdot 8(35 \cdot 8-81 \cdot 8)$ \\
\hline \multirow[t]{3}{*}{$2013 / 14$} & $2-17$ & $107(13)$ & $1867(144)$ & $-65 \cdot 5(-202 \cdot 8-9 \cdot 6)$ & $-18 \cdot 6(-120 \cdot 8-36 \cdot 3)$ & $14(2)$ & 1867 (144) & $\dagger$ & $\dagger$ \\
\hline & $2-6$ & $70(7)$ & $1174(75)$ & $-62 \cdot 8(-267 \cdot 9-27 \cdot 9)$ & $-22 \cdot 5(-182 \cdot 9-46 \cdot 9)$ & $4(1)$ & $1174(75)$ & $\dagger$ & $\dagger$ \\
\hline & $7-17$ & $37(6)$ & $693(69)$ & $-75 \cdot 0(-334 \cdot 3-29 \cdot 5)$ & $-17 \cdot 6(-201 \cdot 0-54 \cdot 0)$ & $10(1)$ & $693(69)$ & $\dagger$ & $\dagger$ \\
\hline \multirow[t]{3}{*}{$2014 / 15$} & $2-17$ & $527(55)$ & $2071(179)$ & $-23 \cdot 2(-69 \cdot 4-10 \cdot 4)$ & $1 \cdot 5(-39 \cdot 2-30 \cdot 3)$ & $210(11)$ & 2071 (179) & $41 \cdot 6(-9 \cdot 3-68 \cdot 8)$ & $60 \cdot 3(23 \cdot 5-79 \cdot 4)$ \\
\hline & $2-6$ & $283(22)$ & $1225(93)$ & $-2 \cdot 6(-66 \cdot 4-36 \cdot 8)$ & $9 \cdot 3(-54 \cdot 3-46 \cdot 7)$ & 69 (3) & $1225(93)$ & $44 \cdot 7(-79 \cdot 4-82 \cdot 9)$ & $54 \cdot 9(-51 \cdot 7-86 \cdot 6)$ \\
\hline & $7-17$ & $244(33)$ & $846(86)$ & $-38 \cdot 2(-112 \cdot 3-10 \cdot 0)$ & $-5 \cdot 6(-68 \cdot 3-33 \cdot 7)$ & $141(8)$ & $846(86)$ & $46 \cdot 8(-12 \cdot 3-74 \cdot 8)$ & $63 \cdot 6(20 \cdot 3-83 \cdot 4)$ \\
\hline \multirow[t]{3}{*}{$2015 / 16$} & $2-17$ & $10(1)$ & $1593(122)$ & $\dagger$ & $\dagger$ & $423(28)$ & $1593(122)$ & $14 \cdot 5(-30 \cdot 8-44 \cdot 1)$ & $42 \cdot 3(7 \cdot 2-64 \cdot 2)$ \\
\hline & $2-6$ & $8(1)$ & $972(63)$ & $\dagger$ & $\dagger$ & $176(8)$ & $972(63)$ & $31 \cdot 3(-46 \cdot 0-67 \cdot 7)$ & $47 \cdot 1(-22 \cdot 5-77 \cdot 2)$ \\
\hline & $7-17$ & $2(0)$ & $621(59)$ & $\dagger$ & $\dagger$ & $247(20)$ & $621(59)$ & $16 \cdot 1(-42 \cdot 6-50 \cdot 6)$ & $35 \cdot 6(-15 \cdot 9-64 \cdot 2)$ \\
\hline \multirow{3}{*}{$2012-2016^{*}$} & $2-17$ & 1116 (99) & $7323(591)$ & $-10 \cdot 9(-38 \cdot 6-11 \cdot 3)$ & $8 \cdot 4(-16 \cdot 6-28 \cdot 0)$ & $1343(67)$ & $7323(591)$ & $40 \cdot 2(22 \cdot 5-53.9)$ & $50 \cdot 7(34 \cdot 3-63 \cdot 0)$ \\
\hline & $2-6$ & $668(45)$ & $4520(307)$ & $0 \cdot 9(-37 \cdot 0-28 \cdot 3)$ & $13 \cdot 0(-23 \cdot 2-38 \cdot 6)$ & $505(23)$ & $4520(307)$ & $34 \cdot 5(-1 \cdot 1-57 \cdot 6)$ & $40 \cdot 9(5 \cdot 1-63 \cdot 2)$ \\
\hline & $7-17$ & $448(54)$ & $2803(284)$ & $-21 \cdot 6(-65 \cdot 7-10 \cdot 8)$ & $2 \cdot 6(-36 \cdot 8-30 \cdot 7)$ & $838(44)$ & $2803(284)$ & $50 \cdot 8(31 \cdot 8-64 \cdot 6)$ & $53 \cdot 8(33 \cdot 5-67 \cdot 9)$ \\
\hline
\end{tabular}

Statisically significant estimates (95\% confidence limits excluding zero) are printed in bold.

* Adjusted for sex, age, month of illness onset and federal state.

$\dagger$ No VE estimate because of a too small number of cases $(<30)$.

$\$$ Adjusted estimates additionally adjusted for season. 
patients tested positive, $811(24 \cdot 5 \%)$ tested positive for A(H1N1)pdm09, $1116(33 \cdot 8 \%)$ for A(H3N2), 46 for unsubtyped influenza A $(1 \cdot 4 \%)$ and $1343(40 \cdot 6 \%)$ for influenza B. Twelve patients had co-infections with two different subtypes of influenza.

Influenza-positive proportions during the seasons 2012/13, 2014/15 and 2015/16 were higher than 30\% (44.6\%, 1443 cases; $31 \cdot 2 \%, 940 ; 32 \cdot 6 \%, 769)$, whereas the positive proportion in $2013 / 14$ was only $7 \cdot 5 \%$ (152).

The proportion of $\mathrm{A}(\mathrm{H} 1 \mathrm{~N} 1) \mathrm{pdm} 09$ of all cases was highest in 2015/16 with $42 \cdot 1 \%$ (324 cases) and below $20 \%$ during the other seasons. Influenza $\mathrm{A}(\mathrm{H} 3 \mathrm{~N} 2)$ was the predominant subtype in 2013/14 (107 cases, $70 \cdot 4 \%$ ) and 2014/15 (527, 56.1\%). Influenza B was predominant in 2012/13 (696, 48.2\%) and 2015/16 (423, $55 \cdot 0 \%)$.

\section{Vaccine effectiveness}

Any influenza

Crude estimate for VE against any influenza across all seasons was $23 \cdot 1 \%(95 \%$ CI: $9 \cdot 4-34 \cdot 7)$, adjusted estimate for VE against any influenza across all seasons was $33 \cdot 0 \%(95 \%$ CI: $24 \cdot 3-40 \cdot 7)$ for all age groups (Table 3). Season-specific VE estimates indicated a preventive effect being statistically significant only for seasons 2012/13 (50.7\%, 95\% CI: 32.5-64.0) and 2015/16 (49.4\%, 95\% CI: 25·7-65.5). Point estimates of VE were higher for age group 7-17 years than for the younger ones, but the differences were not statistically significant. This was also the case in the following subtype-analyses (Table 3).

\section{A(H1N1)pdm09}

Across all seasons, adjusted estimate for VE against laboratory-confirmed $\mathrm{A}(\mathrm{H} 1 \mathrm{~N} 1) \mathrm{pdm} 09$ was $47.0 \%$ (95\% CI: 25·7-62.2) among both age groups. VE estimates across both age groups were $56.7 \%(95 \% \mathrm{CI}$ : $17 \cdot 1-77 \cdot 4)$ in $2012 / 13$ and $60 \cdot 2 \%(95 \%$ CI: $29 \cdot 3-$ $77 \cdot 6$ ) in 2015/16. Whereas estimated VE for $7-$ 17-year-olds was $73 \cdot 2 \%(95 \%$ CI: $22 \cdot 7-90 \cdot 7)$ in $2012 /$ 13 and $86 \cdot 2 \%(95 \%$ CI: $41 \cdot 3-96 \cdot 7)$ in $2015 / 16$, estimates for children aged 2-6 years were $33 \cdot 1 \%(95 \%$ CI: $-52 \cdot 8-70 \cdot 7)$ and $44 \cdot 8 \%(95 \% \mathrm{CI}:-6 \cdot 9-71 \cdot 5)$, respectively. Estimated VE was $9 \cdot 3 \% \quad(95 \% \mathrm{CI}$ : $-62 \cdot 0-49 \cdot 3)$ in $2014 / 15$. A negative point estimate was obtained for children aged 7-17 years in 2014/ 15 (-8.0, 95\% CI: $-173 \cdot 0-57 \cdot 3)$ (Table 3).

\section{A(H3N2)}

Estimated VE against A(H3N2) across all seasons was $8 \cdot 4 \%(95 \%$ CI: $-16 \cdot 6-28 \cdot 0)$. Highest estimate was $30 \cdot 2 \%(95 \% \mathrm{CI}:-7 \cdot 1-54 \cdot 5)$ in season $2012 / 13$. We obtained negative estimates in 2013/14 $(-18.6 \%$, 95\% CI: $-120 \cdot 8-36 \cdot 3)$ among both age groups. In 2014/15, estimated VE was $1 \cdot 5 \%$ (95\% CI: $-39 \cdot 2$ $30 \cdot 3), 9 \cdot 3 \%(95 \% \mathrm{CI}:-54 \cdot 3-46 \cdot 7)$ for children aged 2-6 years and $-5 \cdot 6 \%(95 \% \mathrm{CI}:-68 \cdot 3-33 \cdot 7)$ for $7-$ 17-year-old children (Table 4).

\section{Influenza $B$}

Adjusted VE estimate against laboratory-confirmed influenza $\mathrm{B}$ among all children across all seasons was $50 \cdot 7 \%$ (95\% CI: 34-3-63.0). Highest VE estimates across both age groups were $58 \cdot 6 \%(95 \%$ CI: $34 \cdot 0$ $74 \cdot 0)$ in $2012 / 13$ and $60 \cdot 3 \%$ (95\% CI: $23 \cdot 5-79 \cdot 4)$ in 2014/15. In $2012 / 13$, estimated VE was $65.8 \%(95 \%$ CI: $35 \cdot 8-81 \cdot 8$ ) for $7-17$-year-old children and $41 \cdot 1 \%$ (95\% CI: $-18 \cdot 5-70 \cdot 7)$ for 2- to 6-year-olds. Same patterns were observed in 2014/15. VE was estimated to be $42 \cdot 3 \%$ (95\% CI: $7 \cdot 2-64 \cdot 2)$ in 2015/16 (Table 4).

\section{Sensitivity analysis}

Date of illness onset was missing for $316(13 \cdot 4 \%)$ children. Excluding data of patients with missing date of illness onset, VE against any influenza in 2015/16 was $46 \cdot 4 \%(95 \%$ CI: $17 \cdot 7-65 \cdot 1)$ across both age groups. We observed VE of $39 \cdot 9 \%$ (95\% CI: $-10 \cdot 2-67 \cdot 3)$ in 2- to 6-year-olds and $50 \cdot 3 \%$ (95\% CI: 8·0-73.1) in children aged 7 to 17 years.

\section{DISCUSSION}

We estimated VE against laboratory-confirmed influenza for four influenza seasons among children and adolescents using TND and routine surveillance data from Lower Saxony and Saxony-Anhalt.

We found low to moderate values of VE with an average of $33 \%$ across all seasons. A moderate preventive effect of about $50 \%$ was found for seasons 2012/13 and 2015/16. A strong variation of VE between the seasons and subtypes was observed. VE against $\mathrm{A}(\mathrm{H} 1 \mathrm{~N} 1) \mathrm{pdm} 09$ ranged from $-8 \%(95 \% \mathrm{CI}$ : $-173 \cdot 0-57 \cdot 3$ ) for $7-17$-year-olds in $2014 / 15$ to $86 \cdot 2 \%$ (95\% CI: 41-3-96.7) for 7-17-year-olds in 2015/16. In general, there was a moderate VE against this subtype in 2012/13 and in 2015/16, whereas VE against A (H3N2) was low for every season, ranging from 
$-22 \cdot 5 \%$ (95\% CI: $-182 \cdot 9-46 \cdot 9)$ for 2-6-year-olds in $2013 / 14$ to a maximum of $30 \cdot 2 \%(95 \%$ CI: $-7 \cdot 1-$ 54.5) across both age groups in 2012/13. Against influenza B, highest values were observed for 717-year-old children in 2012/13 $(65.8 \%, 95 \%$ CI: $35 \cdot 8-81 \cdot 8)$ and $2014 / 15(63 \cdot 6 \%, 95 \%$ CI: $20 \cdot 3-83 \cdot 4)$. Low values were observed in 2015/16, when the lowest estimate of $35 \cdot 6 \%$ (95\% CI: - 15.9-64.2) was obtained for 7-17-year-olds. Estimated values for VE are similar compared to the results of other VE studies [13-19].

We conducted sensitivity analysis for 2015/16, excluding 316 children with missing date of illness onset instead of imputing the date. VE of sensitivity analysis did not differ relevantly from main results (46.4\%, 95\% CI: $17 \cdot 7-65 \cdot 1$ vs. $49 \cdot 4 \%, 95 \%$ CI: $25 \cdot 7-$ 65.5 in 2- to 17 -year-olds).

Observed low VE against $\mathrm{A}(\mathrm{H} 3 \mathrm{~N} 2)$, especially in $2014 / 15$, could be explained by a mismatch between circulating strains and vaccine strains. There was a good match for $\mathrm{A}(\mathrm{H} 1 \mathrm{~N} 1) \mathrm{pdm} 09$ through the seasons what coincides with observed moderate VE in 2012/ 13 and 2015/16. Matches were found for influenza B except from 2015/16, when trivalent vaccine did not include a strain of the circulating line [8-11, 22-25].

We found a tendency to higher estimates of VE in children aged 7-17 years for some subtypes and seasons. One possible explanation might be the type of vaccine (LAIV or IIV) itself. Although studies from Finland and the UK showed a preventive effect of LAIV [17, 19, 26, 27], a relatively low or even a lack of preventive effect of LAIV compared with IIV during 2013-2016 was found elsewhere [28]. CDC's Advisory Committee on Immunization Practices (ACIP) therefore voted against the use of LAIV in 2016/17 [28]. We could not stratify directly for vaccine type. If paediatricians complied with the national recommendation, the 2-6-year-olds in our study might have received LAIV more often than older children from 2013/14 to 2015/16. This could be one out of several possible explanations for low VE estimates among this age group but should be interpreted with caution as no coverage data on vaccine type was available. Helmeke et al. examined VE by vaccine type using pooled data from Lower Saxony and Saxony-Anhalt from season 2015/16 and found a lower VE of LAIV, especially against A(H1N1)pdm09, compared with IIV in children [29].

We observed some, statistically not significant, negative point estimates of VE, e.g. $-5 \cdot 6 \%(95 \% \mathrm{CI}$ : $-68 \cdot 3-33 \cdot 7)$ against $\mathrm{A}(\mathrm{H} 3 \mathrm{~N} 2)$ for $7-17$-year-old children in 2014/15. Due to the formula VE $=1-O R$, the estimate was between $0 \%$ and $100 \%$ if cases had a lower odds for being vaccinated than controls, and below zero per cent if this was not the case. Even estimates below $-100 \%$ were possible. Besides random variation, there are further hypotheses explaining the occurrence of negative VE, namely the antigenic distance hypothesis and repeat vaccination effects, as described elsewhere [30, 31]. Skowronski et al. found low VE against A(H3N2) in Canada in 2014/15 and mentioned repeated vaccination effects, variation of virus' genome and a mismatch between wild strain and vaccine strain as possible explanation [32, 33].

Nevertheless, there might be some biases and methodological issues that have to be addressed.

Firstly, information bias, particularly misclassification error, leading to underestimation of $\mathrm{VE}$, has to be taken into account. Misclassification of cases and controls and associated underestimation of VE is negligible in TND when using high sensitive and specific test methods [34, 35]. Hence, this kind of bias can be neglected in our study, as we used highly sensitive and specific RT-PCR for diagnosis of laboratoryconfirmed influenza.

Relating to the exposure collected by the questionnaire, particularly vaccination status and date, this information could have been incorrect, as patients might not have remembered vaccination correctly. When assessing this kind of misclassification error, it has to be considered that in Germany, though there is free choice of physician, people usually attend only one and the same general physician or paediatrician. If the paediatrician who had provided the vaccine before was the same paediatrician who took the sample, he or she should have reported vaccination status correctly.

Secondly, the occurrence of selection bias has to be taken into account. In Germany, the Standing Committee on Vaccination does not recommend influenza vaccination for children in general, but for people with underlying chronic diseases [2]. We expect that children with underlying chronic conditions were overrepresented in our study because they were more likely to consult a paediatrician and thus to be sampled due to their chronic diseases. Nevertheless, there are no hints for a poorer VE in people with underlying chronic disease. That is why we do not think that this substantially affects our results.

Additionally, another kind of selection bias has to be considered. In principal, the paediatricians were asked to select the patients randomly and not according to further criteria besides the clinical case definition of ARI. However, it cannot be excluded that 
vaccinationstatus of the patient or the severity of the disease might have influenced the indication for swabbing in some cases. However, the proportion of vaccinated children was quite low in our sample and very severe cases would rather have attended hospital, not the paediatrician. Thus, as hospital patients represent only a minority of our sample, it is unlikely that this kind of bias has serious impact on our results.

We conclude that there might be several biases that can influence the VE estimates and thus might explain the low estimates, but none of these biases should have substantial effects on our results. However, for the future it is planned to gain more information about underlying chronic diseases, to better address selection bias.

As the study is based on secondary data, we could not obtain additional data on further details of vaccination or confounders. For instance, we had no information on vaccine type, number of received doses and whether the children had received the vaccine for the first time. We added these aspects to our surveillance questionnaire so that we will have this information for future analyses. The main confounders considered in this analysis were month of illness onset, age, sex and season. Not surprisingly, the season had an effect on VE estimates, as the predominating virus subtypes and the vaccine composition differed between the seasons, leading to a better or poorer match between circulating viruses and vaccine viruses. Month of illness onset had an impact on VE estimates as well. We chose February as reference as this month is the peak time of influenza seasons. The risk of an infection with influenza viruses decreased with increasing distance of time from February. Impact of age, federal state and sex varied between the seasons and subtypes.

We accounted for random variation by providing CIs for VE estimates. Many season-specific CIs included an effect of zero per cent. CIs were quite wide because the vaccination coverage of children in the study population was below $10 \%$. Over the study period, there were no changes of the vaccination recommendations for children and thus the vaccination coverage in our study population did not change substantially either. The higher coverage in the older children might be due to the fact that some chronic diseases manifest with increasing age. It would also be conceivable, that parents' experience with influenza and influenza-like illness in their children changes over time and that this aspect plays a role when deciding to get the children vaccinated. The low coverage made it difficult to gain sufficiently large sample sizes for statistically significant estimates. However, compared with similar studies, our sample sizes per season were rather high, especially because we pooled data from two federal states. The primary purpose of the surveillance systems does not justify to further increase sample size. Thus, if higher precision and smaller CIs are required, results from several studies should be brought together by means of meta-analysis (see e.g. Belongia et al., 2016 [18]).

A significant preventive effect of the vaccine against any influenza was found for two out of four observed influenza seasons (2012/13 and 2015/16). A lack of preventive effect, low or even moderate VE estimates, e.g. $49 \cdot 4 \%$ against any influenza in 2015/16, could be problematic when communicating benefits of influenza vaccination to the population. Such values might not help increase vaccination uptake or encourage personal decisions of getting vaccinated. Therefore, communication of VE estimates requires caution. A VE of $50 \%$ should not be confused with 50 out of 100 vaccinated people getting infected with influenza anyway. It needs to be communicated that it still means that the odds of getting ill for the vaccinated ones' is half of the odds of people who did not receive the vaccine. Even if a better evidence of VE might encourage more people to get vaccinated, in long-term, it might be helpful to communicate that influenza vaccine does not have $100 \%$ effectiveness.

Although RCT is the best design to measure vaccine's efficacy [12], observational studies are needed to measure influenza VE in the field. For this purpose, TND is a commonly used method and produces acceptable estimates for true VE [34, 36].

In conclusion, we found a strong variation of vaccines' preventive effect against laboratory-confirmed influenza between the observed seasons and the different subtypes. High values of VE were found against A (H1N1)pdm09, especially in 7-17-year-olds, e.g. $86 \cdot 2 \%$ (95\% CI: $41 \cdot 3-96 \cdot 7)$ in $2015 / 16$ and $73 \cdot 2 \%$ (95\% CI: $22 \cdot 7-90 \cdot 7)$ in 2012/13. Low estimates were observed against $\mathrm{A}(\mathrm{H} 3 \mathrm{~N} 2)$ in any season, e.g. $1 \cdot 5 \%$ (95\% CI: $-39 \cdot 3-30 \cdot 3)$ in $2014 / 15$ and against $A$ (H1N1)pdm09 in 2014/15 when observed VE across both age groups was $9 \cdot 3 \%(95 \% \mathrm{CI}$ : $-62 \cdot 0-49 \cdot 3)$. With regard to the different circulation of influenza virus subtypes during different seasons, low numbers of cases and mentioned explanations for possible underestimation of VE in TND have to be considered here.

\section{ACKNOWLEDGEMENTS}

We would like to thank the participating sentinelpractitioners and clinicians for their contributions and 
the staff of the laboratories of the Governmental Institute of Public Health of Lower Saxony and the State Agency for Consumer Protection of SaxonyAnhalt for their excellent technical assistance.

\section{DECLARATION OF INTEREST}

None.

\section{REFERENCES}

1. World Health Organization (WHO). Influenza (http:// www.who.int/immunization/topics/influenza/en/). Accessed 7 February 2017.

2. Robert Koch-Institut. Empfehlungen der Ständigen Impfkommission (STIKO) am RKI Stand: August 2016 (recommendations of standing committee on vaccination at RKI, August 2016). Epidemiologisches Bulletin 2016; 34: 301-340.

3. Robert Koch-Institut. Empfehlungen der Ständigen Impfkommission (STIKO) am RKI Stand: August 2013 (recommendations of standing committee on vaccination at RKI, August 2013). Epidemiologisches Bulletin 2013; 34: 313-344.

4. Robert Koch-Institut. Stellungnahme der STIKO zur Anwendung von Influenza-Lebendimpfstoffen bei Kindern in der Saison 2016/2017 (standing committee on vaccination comments on the use of life attenuated influenza vaccines in children during season 2016/17). Epidemiologisches Bulletin 2016; 39: 442-443.

5. Falkenhorst G, et al. Background paper to the recommendation for the preferential use of live-attenuated influenza vaccine in children aged 2-6 years in Germany. Bundesgesundheitsblatt 2013; 56: 1557-1564.

6. Grohskopf LA, et al. Prevention and control of seasonal influenza with vaccines. MMWR Recommendations and Reports 2016; 65: 1-54.

7. Paul Ehrlich-Institut. PEI Influenza (saisonal): Informationen $\mathrm{zu}$ den aktuellen Grippeimpfstoffen (Information on the current influenza vaccines) (http:// www.pei.de/DE/infos/fachkreise/impfungen-impfstoffe/ influenza-grippeimpfstoffe-saisonal/influenza-grippeimp fstoffe-node.html). Accessed 7 January 2017.

8. World Health Organization. Recommended composition of influenza virus vaccines for use in the 2012-2013 northern hemisphere influenza season (http://www.who.int/ influenza/vaccines/virus/recommendations/2012_13_north/ en/). Accessed 4 September 2016.

9. World Health Organization. Recommended composition of influenza virus vaccines for use in the 2013-14 northern hemisphere influenza season (http://www. who.int/influenza/vaccines/virus/recommendations/2013 14_north/en/). Accessed 4 September 2016.

10. World Health Organization. Recommended composition of influenza virus vaccines for use in the 2014-2015 northern hemisphere influenza season (http://www.who. int/influenza/vaccines/virus/recommendations/2014_15_ north/en/). Accessed 4 September 2016.
11. World Health Organization. Recommended composition of influenza virus vaccines for use in the 2015-2016 northern hemisphere influenza season (http://www.who. int/influenza/vaccines/virus/recommendations/2015_16_ north/en/). Accessed 4 September 2016.

12. Weinberg GA, Szilagyi PG. Vaccine epidemiology: efficacy, effectiveness, and the translational research roadmap. The Journal of Infectious Diseases 2010; 201: 1607-1610.

13. Kissling E, et al. Influenza vaccine effectiveness estimates in Europe in a season with three influenza type/subtypes circulating: the I-MOVE multicentre case-control study, influenza season 2012/13. Eurosurveillance 2014; 19: 20701

14. Kissling E, Valenciano M. Early influenza vaccine effectiveness results 2015-16: I-MOVE multicentre casecontrol study. Eurosurveillance 2016; 21: 30134.

15. Sullivan SG, et al. Pooled influenza vaccine effectiveness estimates for Australia, 2012-2014. Epidemiology and Infection 2016; 144: 2317-2328.

16. Kelly $\mathbf{H}$, et al. Estimation of influenza vaccine effectiveness from routine surveillance data. PLOS ONE. 2009; 4: e5079.

17. Pebody R, et al. Effectiveness of seasonal influenza vaccine in preventing laboratory-confirmed influenza in primary care in the United Kingdom: 2014/15 end of season results. Eurosurveillance 2015; 20: 30013.

18. Belongia EA, et al. Variable influenza vaccine effectiveness by subtype: a systematic review and meta-analysis of test-negative design studies. The Lancet Infectious Diseases. 2016; 16: 942-951.

19. Helmeke C, et al. Effectiveness of the 2012/13 trivalent live and inactivated influenza vaccines in children and adolescents in Saxony-Anhalt, Germany: a test-negative case-control study. PLOS ONE 2015; 10: e0122910.

20. Haas W. Influenza: Prävention, Diagnostik, Therapie und öffentliche Gesundheit (Prevention, Diagnostic, Therapy and Public Health). München: Elsevier Urban \& Fischer, 2009, p. 24.

21. Swart E, et al. Gute Praxis Sekundardatenanalyse (GPS): Leitlinien und Empfehlungen (good practice of secondary data analysis (GPS): guidelines and recommendations). Gesundheitswesen 2015; 77: 120-126.

22. Robert Koch-Institut. Bericht zur Epidemiologie der Influenza in Deutschland Saison 2012/13 (Epidemiological report on influenza in Germany season 2012/13) (https://influenza.rki.de/Saisonberichte/ 2012.pdf). Accessed 20 August 2017.

23. Robert Koch-Institut. Bericht zur Epidemiologie der Influenza in Deutschland Saison 2013/14 (Epidemiological report on influenza in Germany in 2013/14) (https://influenza.rki.de/Saisonberichte/2013. pdf). Accessed 20 August 2017.

24. Robert Koch-Institut. Bericht zur Epidemiologie der Influenza in Deutschland Saison 2014/15 (Epidemiological report on influenza in Germany season 2014/15) (https://influenza.rki.de/Saisonberichte/ 2014.pdf). Accessed 20 August 2017.

25. Robert Koch-Institut. Bericht zur Epidemiologie der Influenza in Deutschland Saison 2015/16 
(Epidemiological report on influenza season 2015/16) (https://influenza.rki.de/Saisonberichte/2015.pdf).

Accessed 20 August 2017.

26. Nohynek H, et al. Effectiveness of the live attenuated and the inactivated influenza vaccine in two-year-olds a nationwide cohort study Finland, influenza season 2015/16. Eurosurveillance 2016; 21: 30346.

27. Pebody R. Influenza vaccine effectiveness in adults and children in primary care in the UK: provisional end-of-season results 2015-16 (https://www.gov.uk/government/uploads/system/uploads/attachment_data/file/ 530756/Influenza_vaccine_effectiveness_in_primary_ care_in_children.pdf). Accessed 20 October 2016.

28. Centers for Disease Control and Prevention (CDC). ACIP votes down use of LAIV for 2016-2017 flu season (http://www.cdc.gov/media/releases/2016/s0622-laiv-flu. html). Accessed 20 October 2016.

29. Landesamt für Verbraucherschutz Sachsen-Anhalt. Effektivität der Influenza-Impfstoffe bei Kindern in Sachsen-Anhalt und Niedersachsen 2015/16 (Effectiveness of the influenza vaccine in children in Saxony-Anhalt and Lower Saxony 2015/16) (http:// www.verbraucherschutz.sachsen-anhalt.de/fileadmin/ Bibliothek/Politik_und_Verwaltung/MS/LAV_Verbr aucherschutz/hygiene/influenza/Effektivitaet_der_Inf luenzaimpfstoffe_2015-16.pdf). Accessed 12 February 2017.
30. Belongia EA, et al. Repeated annual influenza vaccination and vaccine effectiveness: review of evidence. Expert Review of Vaccines 2017; 16: 1-14.

31. Smith DJ, et al. Variable efficacy of repeated annual influenza vaccination. Proceedings of the National Academy of Sciences of the United States of America 1999; 96: 14001-14006.

32. Skowronski DM, et al. A perfect storm: impact of genomic variation and serial vaccination on Low influenza vaccine effectiveness during the 2014-2015 season. Clinical Infectious Diseases 2016; 63: 21-32.

33. Skowronski DM, et al. Interim estimates of 2014/15 vaccine effectiveness against influenza $\mathrm{A}(\mathrm{H} 3 \mathrm{~N} 2)$ from Canada's Sentinel Physician Surveillance Network, January 2015. Eurosurveillance 2015; 20: pii=21022.

34. Jackson ML, Rothman KJ. Effects of imperfect test sensitivity and specificity on observational studies of influenza vaccine effectiveness. Vaccine 2015; 33: 1313-1316.

35. Orenstein EW, et al. Methodologic issues regarding the use of three observational study designs to assess influenza vaccine effectiveness. International Journal of Epidemiology 2007; 36: 623-631.

36. De Serres G, et al. The test-negative design: validity, accuracy and precision of vaccine efficacy estimates compared to the gold standard of randomised placebocontrolled clinical trials. Eurosurveillance 2013; 18: pii $=20585$. 